\title{
High resolution evaluation of various cochlear implant electrodes' scalar position by 3T MRI
}

\section{Riemann}

Bielefeld University

\section{LU Scholtz}

Bielefeld University

HB Gehl

Bielefeld University

H Sudhoff

Bielefeld University

I. Todt ( $\square$ todt@gmx.net )

Bielefeld University

\section{Research Article}

Keywords: MRI, cochlear implant, electrode position, quality control

Posted Date: April 9th, 2021

DOI: https://doi.org/10.21203/rs.3.rs-397570/v1

License: (c) (i) This work is licensed under a Creative Commons Attribution 4.0 International License. Read Full License 


\section{Abstract}

Objective: The estimation of scalar electrode position is a central point of quality control during the cochlear implant procedure. Ionic radiation is a disadvantage of commonly used radiologic estimation of electrode position. Recent developments in the field of cochlear implant magnets, implant receiver magnet position, and MRI sequence usage allow the postoperative evaluation of inner ear changes after cochlear implantation.

The aim of the present study was to evaluate the position of lateral wall and modiolar cochlear implant electrodes using 3 T MRI scanning.

Material and Methods: In a prospective study, we evaluated 20 patients (10x Med-El Flex 28; 5x HFMS AB and $5 \times$ Slim J AB) with a 3T MRI and a T2 2D Drive MS sequence (voxel size: $0.3 \times 0.3 \times 0.9 \mathrm{~mm}$ ) for the estimation of the intracochlear position of the cochlear implant electrode.

Results: In all cases, MRI allowed a determination of the electrode position in relation to the basilar membrane. This observation made the estimation of 19 scala tympani electrode positions and a single case of electrode translocation possible.

Conclusion: 3T MRI scanning allows the estimation of lateral wall and modiolar electrode intracochlear scalar positions.

\section{Introduction}

Quality control in modern medicine plays an increasingly important role and follows structured evaluation pathways. ${ }^{1}$ In otology, multiple methods and stages of quality control have been discussed and implemented. ${ }^{2}$

A central point in cochlear implantation quality control is based on the estimation of electrode inside the cochlea, originally performed with a $2 \mathrm{D} \mathrm{X}$-ray. ${ }^{3} \mathrm{~A}$ further central point in the development and establishment of cochlear implant quality control was based on the work of Aschendorff et al, ${ }^{4}$ who provided a 3D impression of the electrode position for the first time by differentiating between scala tympani (ST) position, scala vestibuli (SV) position, and translocation between both scalae. The importance of this finding was underlined by the correlation of the electrode position and speech perception values showing significant correlation. ${ }^{5-7}$ Based on these findings, quality control was not only a tool for the evaluation of surgeons' abilities in the theater, ${ }^{8}$ but a tool for the evaluation of different electrodes as well. ${ }^{9}$

The radiological in vivo results were confirmed by temporal bone findings ${ }^{10}$ and histological comparisons. ${ }^{11}$ 
The disadvantages of ionic radiation led to the evaluation of electrophysiological tools for the estimation of electrode translocations performed by a neural response telemetry (NRT) ratio ${ }^{12}$ with limitations of electrode design and manufacturer brand.

The technique of an overlay of the postoperative digital volume tomography/ computed tomography (DVT/CT) and the preoperative magnet resonance imaging (MRI) integrated MRI for the first time into the evaluation of electrode position, ${ }^{13}$ but reconstruction was time-consuming, and the use of postoperative ionic radiation persisted.

With the development of a new generation of cochlear implant magnets, knowledge about the importance of implant positioning, ${ }^{14-16}$ and intrascanning- head position, ${ }^{17}$ a pain-free evaluation of the cochlea after cochlear implantation ${ }^{18}$ became possible without problems of magnetic artifacting.

Initial evaluations of MRI scanning showed the general possibility of estimating electrode translocation of perimodiolar electrodes, ${ }^{19}$ but limitations to visual resolution persisted. ${ }^{20}$ Model testing allowed the optimization of MRI sequence patterns to solve this problem. ${ }^{21}$

The aim of the present study is to show that an estimation of electrode translocation can be performed with different electrode designs using a 3T MRI.

\section{Materials And Methods}

In this prospective study, 20 patients received cochlear implantation between January and December 2020. In all cases, the cochlear implant receiver magnet was positioned $8 \mathrm{~cm}$ or more behind the external auditory canal. ${ }^{15}$ In addition to regular postoperative DVT for the estimation of electrode position, a 3T MRI scanning was performed.

In this series, no inner ear anomalies, ossification, or tumors were included. In 10 cases, a Synchrony $1 / 2$ Flex 28 (Med-El, Innsbruck, Austria) was implanted. In 5 cases, High Focus Midscalar (HFMS) 3D Advanced Bionics and SlimJ 3D Advanced Bionics (Advanced Bionics, Stäfa, Switzerland) devices were implanted. Individual data are shown in Tab.1.

MRI: Achieva 3 T, Philips Medical System, Best, Netherlands

Sequence: T2 2D Drive MS, voxel size $0.3 \times 0.3 \times 0.9 \mathrm{~mm}$; FOV $150 \times 150$, TE $100 \mathrm{~ms}$ TR 3000, TSE tact 17 , multi shot, flip angle $90^{\circ}$, refocus control 120 , metric 512; NSA 5, foldover: AP

DVT: New TOM VGI, Verona, Italy

Parameters: FOV $15 \times 15 \mathrm{~cm}, 10,48 \mathrm{mAS}-20,52 \mathrm{mAS}, \mathrm{KV} 110,360^{\circ}$ followed by $2 \mathrm{D}$ and 3D reconstruction at an external workstation (NNT, main station).

The MSCT Toshiba Aquilion 80 protocol was: slice thickness 0,5 mm, KV 120, MA 200, rot.time 0,75 
Electrode position in terms of scalar location (DVT/ CT and MRI) was evaluated independently by two surgeons and a neuroradiologist.

Patients gave written informed consent. The study was approved by the institutional review board (IRB) (HNO- KliBi, 001/2020) and the ethical board of the University Münster (2019-135-f-S)

\section{Results}

Evaluation of cochlear implant electrode by 3 T MRI showed 2 visual indicators of electrode position in the axial plain. For the basal turn, a diminishing signal indicates the electrode localisation. At the lateral portion of the cochlea, each electrode was positioned depending on its design: either laterally, without any further lateral T2 signal (Fig. 1a and 1b), or more medially positioned with a T2 signal (Fig. 3). Lateral diminishing signal varied depending on electrode design: Flex 28 was round, and SlimJ laterally flatter. As a second indicator, a basilar membrane was visible. This basilar membrane diminishing signal allowed definitive estimation of the electrode location in the scala tympani or scala vestibuli.

At the first turn, a design-specific difference of the diminishing signal of the electrodes was obvious. We were able to differentiate between a round signal diminishing and a flatter signal diminishing (Fig. 2a and Fig. 2b). The basilar membrane signal (Fig. 2a and 2 b) and the localization of the fluid signal diminishing indicate scala tympani positions at the first turn for these two types of lateral wall electrodes.

Modiolar electrode characteristics allowed us to visualize a lateral T2 signal from the electrode at the basal turn and a basilar membrane pattern (Fig. 3 and 4). This basilar membrane pattern allowed us to locate the electrode in the scala tympani definitely.

At the first turn, the estimation of the electrode position depends on the estimation of the basilar membrane and the diminishing localisation of the T2 signal by the electrode. In Fig. 5, diminishing occurred above the T2 signal and the basilar membrane signal, indicating fluid. This indicated the pattern of an electrode translocated into the scala vestibuli. Fig.7 of the same patient (No.10) confirms the irregular high position of the electrode in the first turn. In Fig. 3 and 4, the signal diminished above the electrode and the basilar membrane, indicating an electrode position in the scala tympani. Fig.6a and $b$ are exemplary DVT of regular electrode positions at the floor of the basal and the first turn.

Independent evaluation of electrode positions by one surgeon and a neuroradiologist using DVT/ CT and MRI confirmed the estimated MRI positions in all and in DVT/CT cases in almost all cases (Test: Quadratic-Weighted Cohen's $\mathrm{k}$ with 95\% confidence intervals; MRI: Kappa $=1.000$; SE of kappa $=0.000$, $95 \%$ confidence interval: From 0.704 to $1.00 ;$ DVT: Kappa $=0.898 ; \mathrm{SE}$ of kappa $=0.099,95 \%$ confidence interval: From 0.704 to 1.00$)$.

Limitations of the study persist in terms of MRI resolution. Although voxel size of $0.3 \mathrm{~mm}$ could be used, a free reconstruction related to a non 3D sequence (building of iso voxel) was not possible. A $3 \mathrm{D}$ MRI sequence commonly generates artifacts, which do not allow a visualization of the IAC and cochlea. 


\section{Discussion}

Quality control plays an important role in the structuration of medical pathways. ${ }^{1}$ Even in otology, quality control is well known. ${ }^{2}$ In the surgical portion of a cochlear implant procedure, electrode placement is of high importance for the audiological outcome. ${ }^{7}$ The value of different radiological techniques for estimation of electrode positions lies in the evaluation of the surgeon ability of positioning and the properties of the electrode design to ensure the desired target position is met.

Different radiological techniques have been shown to determine electrode position in $2 D^{3}$ or $3 D^{4}$ patterns. The combination of postoperative DVT with an overlapping preoperative MRI allows electrode positions to be estimated relative to the MRI. ${ }^{13}$

The disadvantage of all these techniques is the occurrence of ionic radiation. Electrophysiological techniques such as the NRT ratio ${ }^{12}$ show promising results, but have limitations in terms of electrode design and depend on tissue properties or the intracochlear neural state.

Our technique demonstrates an option for estimating the electrode position without ionic radiation. This is clinically important, especially in children.

This technical refinement allows visual determination of the basilar membrane for the first time, underlining the important development of visual resolution in comparison to previous studies. ${ }^{19,20}$ Our findings qualify the estimation of translocations for lateral wall electrodes and modiolar electrodes.

The usage of a commonly used 3T MRI scanner underlines the option for regular usage of our technique in the clinical routine.

However, limitations persist for the estimation of insertion depths, anomalies, and specific electrode conditions such as tip folding.

\section{Conclusion}

3T MRI scanning allows the estimation of lateral wall and modiolar electrode scalar positions.

\section{Declarations}

\section{Acknowledgement:}

We would like to thank S. Trees for her excellent radiological support.

\section{Statement of ethics:}

The data used to support this study's findings are available from the corresponding author upon request. 
The study was approved by the institutional review board of the Klinikum Bielefeld, Germany (IRB-klibiHNO-2020/001) and the ethical board of the University Münster (2019-135-f-S). Patients gave written informed consent for the use of their clinical records in this study. The study was conducted in accordance with the World Medical Association Declaration of Helsinki.

\section{Disclosure Statement:}

The corresponding author received grants and travel support in the last five years from Advanced Bionics, MED-El and Cochlear.

\section{Data availability statement:}

The datasets generated during and/or analysed during the current study are available from the corresponding author on reasonable request.

\section{Funding sources:}

No funding

\section{Author Contributions}

C. Riemann, Assistant, evaluation of data, analysis of scans

LU Scholtz, Consultant, cowriting

H.B. Gehl , Head of Bielefeld Radiology, discussion of radiological results, cowriting

H. Sudhoff , Head of Bielefeld Otola. Department, cowriting

I. Todt, Consultant, surgeon, idea, writing

\section{References}

1. Zamboni $\mathrm{K}$, Baker $\mathrm{U}$, Tyagi $\mathrm{M}$, et al. How and under what circumstances do quality improvement collaboratives lead to better outcomes? A systematic review. Implement Sci. 2020;15(1):27. doi:10.1186/s13012-020-0978-z

2. Neudert M, Zahnert T. Tympanoplasty-news and new perspectives. GMS Curr Top Otorhinolaryngol Head Neck Surg. 2017;16. doi:10.3205/cto000146

3. Marsh MA, Xu J, Blamey PJ, et al. Radiologic evaluation of multichannel intracochlear implant insertion depth. Am J Otol. 1993;14(4):386-391.

4. Aschendorff A, Kubalek R, Turowski B, et al. Quality control after cochlear implant surgery by means of rotational tomography. Otol Neurotol. 2005;26(1):34-37. doi:10.1097/00129492-20050100000007 
5. Aschendorff A, Kromeier J, Klenzner T, Laszig R. Quality control after insertion of the nucleus contour and contour advance electrode in adults. Ear Hear. 2007;28(2 Suppl):75S-79S.

doi:10.1097/AUD.0b013e318031542e

6. Finley CC, Holden TA, Holden LK, et al. Role of electrode placement as a contributor to variability in cochlear implant outcomes. Otol Neurotol. 2008;29(7):920-928.

doi:10.1097/MAO.0b013e318184f492

7. Holden LK, Finley CC, Firszt JB, et al. Factors affecting open-set word recognition in adults with cochlear implants. Ear Hear. 2013;34(3):342-360. doi:10.1097/AUD.0b013e3182741aa7

8. Aschendorff A, Klenzner T, Arndt S, et al. Insertionsergebnisse von Contour ${ }^{\mathrm{TM}}$ - und Contour-Advance ${ }^{\mathrm{TM}}$ Elektroden: Gibt es individuelle Lernkurven? Insertion results for Contour ${ }^{\mathrm{TM}}$ and Contour Advance ${ }^{\mathrm{TM}}$ electrodes: are there individual learning curves? HNO. 2011;59(5):448-452. doi:10.1007/s00106-0112319-7

9. Boyer E, Karkas A, Attye A, et al. Scalar localization by cone-beam computed tomography of cochlear implant carriers: a comparative study between straight and periomodiolar precurved electrode arrays. Otol Neurotol. 2015;36(3):422-429. doi:10.1097/MA0.0000000000000705

10. Bartling SH, Gupta R, Torkos A, et al. Flat-panel volume computed tomography for cochlear implant electrode array examination in isolated temporal bone specimens. Otol Neurotol. 2006;27(4):491498. doi:10.1097/01.mao.0000194816.15298.50

11. Marx M, Risi F, Escudé $B$, et al. Reliability of cone beam computed tomography in scalar localization of the electrode array: a radio histological study. Eur Arch Otorhinolaryngol. 2014;271(4):673-679. doi:10.1007/s00405-013-2448-6.

12. Mittmann P, Ernst A, Todt I. Intraoperative electrophysiologic variations caused by the scalar position of cochlear implant electrodes. Otol Neurotol. 2015;36(6):1010-1014. doi:10.1097/MA0.0000000000000736

13. Sipari S, Iso-Mustajärvi M, Löppönen $\mathrm{H}$, Dietz A. The insertion results of a mid-scala electrode assessed by MRI and CBCT image fusion. Otol Neurotol. 2018;39(10):e1019-e1025. doi:10.1097/MA0.0000000000002045

14. Walton J, Donnelly NP, Tam YC, et al. MRI without magnet removal in neurofibromatosis type 2 patients with cochlear and auditory brainstem implants. Otol Neurotol. 2014;35(5):821-825. doi:10.1097/MA0.0000000000000330

15. Todt I, Rademacher G, Mittmann P, et al. MRI artifacts and cochlear implant positioning at $3 \mathrm{~T}$ in vivo. Otol Neurotol. 2015;36(6):972-976. doi:10.1097/MA0.0000000000000720

16. Carlson ML, Neff BA, Link MJ, et al. Magnetic resonance imaging with cochlear implant magnet in place: safety and imaging quality. Otol Neurotol. 2015;36(6):965-971. doi:10.1097/MA0.0000000000000666

17. Ay N, Gehl HB, Sudhoff H, Todt I. Effect of head position on cochlear implant MRI artifact. Eur Arch Otorhinolaryngol. Published online Sep 9, 2020. doi:10.1007/s00405-020-06349-1. 
18. Todt I, Tittel A, Ernst A, Mittmann P, Mutze S. Pain-Free 3T MRI scans in cochlear implantees. Otol Neurotol. 2017;38(10):e401-e404. doi:10.1097/MA0.0000000000001569

19. Stratmann A, Mittmann P, Rademacher G, et al. MRI-Based estimation of scalar cochlear-implant electrode position. Biomed Res Int. Published online Oct 17, 2017. doi:10.1155/2017/6372704

20. Tek F, Müller S, Boga E, et al. 3T MRI-based estimation of scalar cochlear implant electrode position. Acta Otorhinolaryngol Ital. 2019;39(4):269-273. doi:10.14639/0392-100X-2309

21. Todt I, Riemann C, Gehl HB, Sudhoff Cochlear model for the evaluation of MRI sequences and cochlear implant electrode pattern at 3T. Acta Otola, 2021 (submitted).

\section{Table}

Tab.1 Individual surgery date, electrode and position. 


\begin{tabular}{|c|c|c|c|}
\hline No & Surgery & Electrode & Position \\
\hline 20 & 5.3 .20 & Slim J & ST \\
\hline 19 & 4.5 .20 & Flex 28 & ST \\
\hline 18 & 27.5 .20 & Flex 28 & ST \\
\hline 17 & 13.8.20 & Flex 28 & ST \\
\hline 16 & 18.8.20 & Flex 28 & ST \\
\hline 15 & 22.9.20 & Flex 28 & ST \\
\hline 14 & 13.10 .20 & Flex 28 & ST \\
\hline 13 & 15.10 .20 & HFMS & ST \\
\hline 12 & 29.10 .20 & HFMS & ST \\
\hline 11 & 6.11 .20 & HFMS & ST \\
\hline 10 & 15.11 .20 & HFMS & Translocation \\
\hline 9 & 17.11 .20 & Slim J & ST \\
\hline 8 & 26.11 .20 & HFMS & ST \\
\hline 7 & 1.12 .20 & Flex 28 & ST \\
\hline 6 & 3.12 .20 & Flex 28 & ST \\
\hline 5 & 15.12 .20 & Flex 28 & ST \\
\hline 4 & 16.12 .20 & Slim J & ST \\
\hline 3 & 18.12.20 & Slim J & ST \\
\hline 2 & 8.1 .21 & Flex 28 & ST \\
\hline 1 & 28.1 .21 & Slim J & ST \\
\hline
\end{tabular}

Figures 
(B)
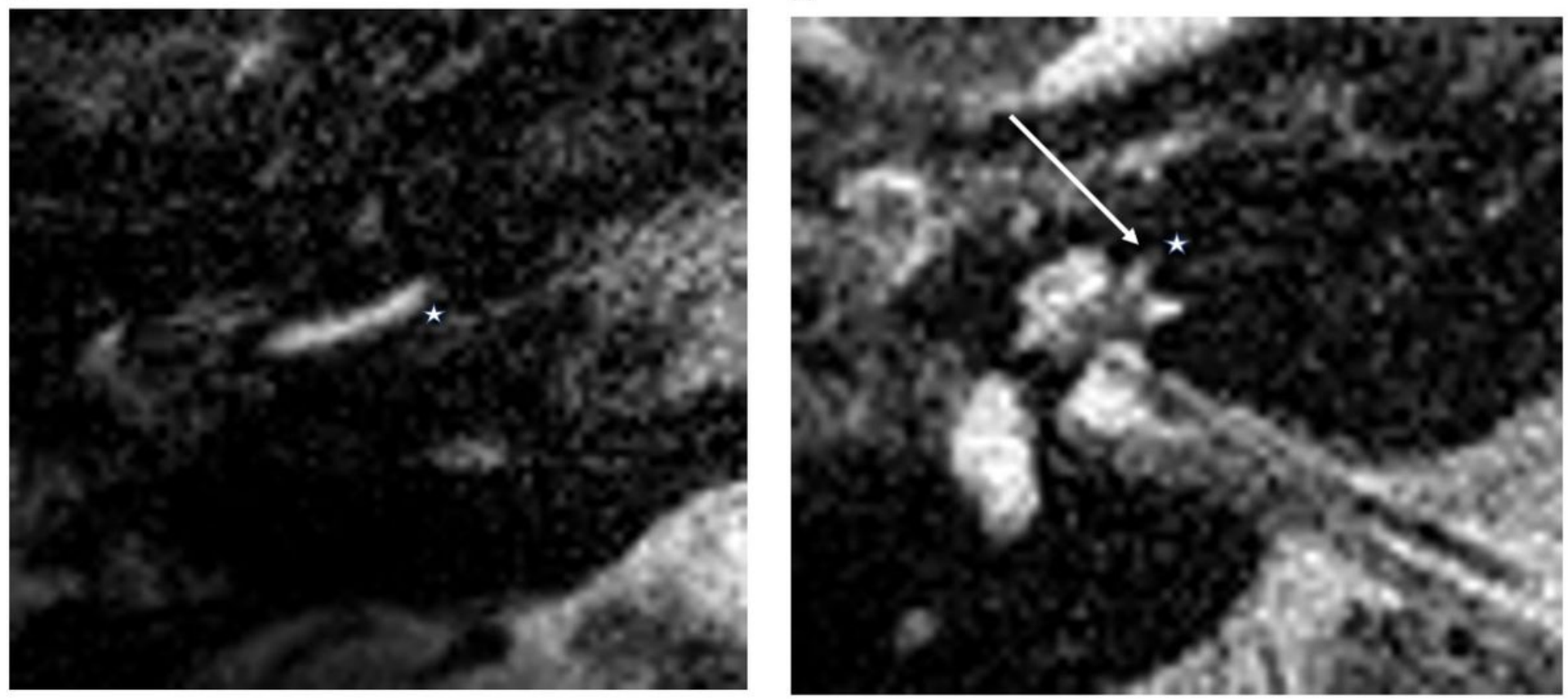

\section{Figure 1}

Straight Electrodes, Basal Turn (A) Flex 28, (B) SlimJ. Star represents diminishing electrode signal, arrow represents basilar membrane.

(A)

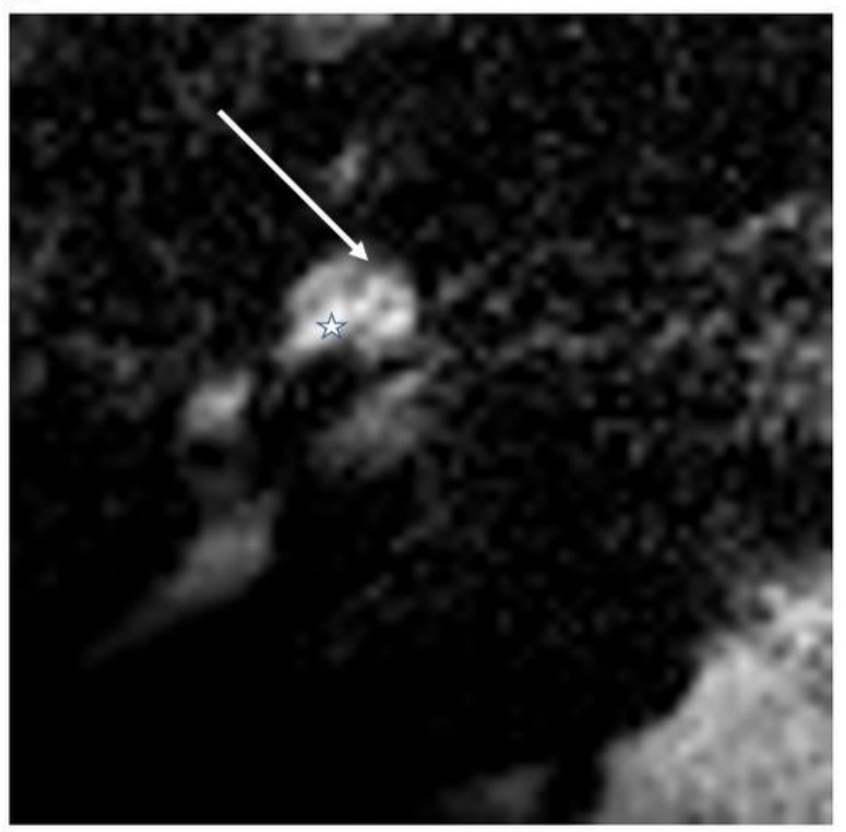

(B)

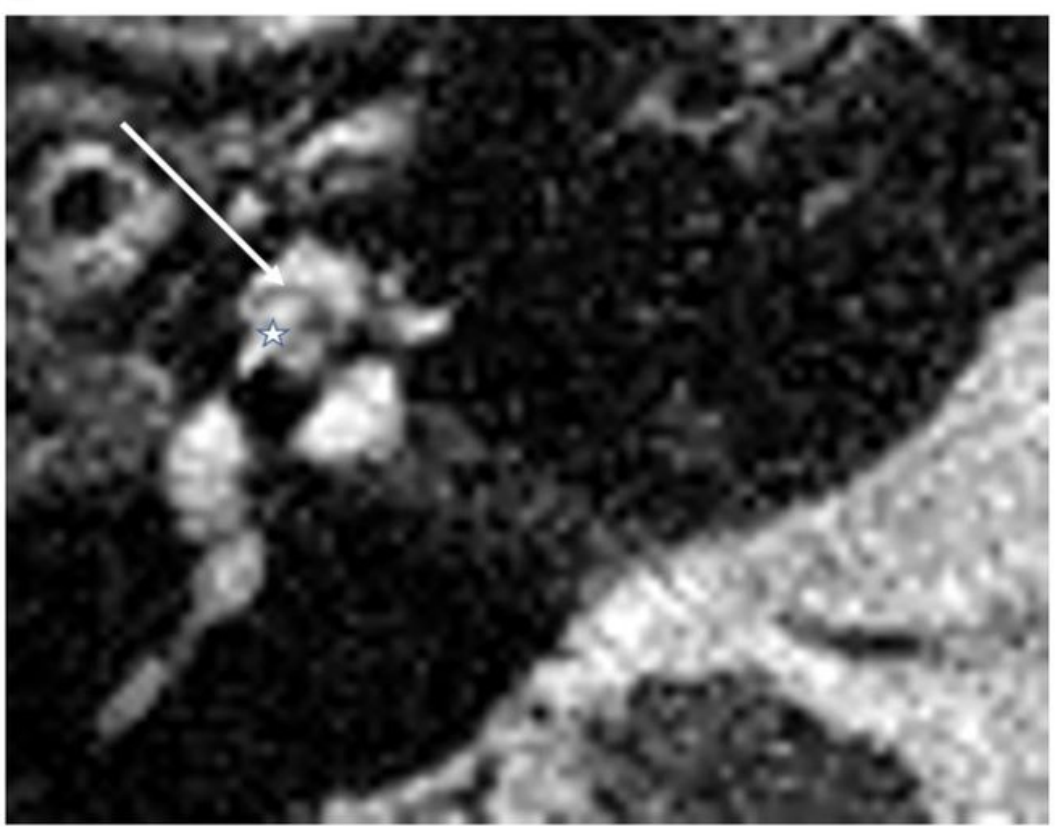

\section{Figure 2}

Straight Electrodes, First Turn (A) Flex 28, (B) SlimJ. Star represents diminishing electrode signal, arrow represents basilar membrane. 


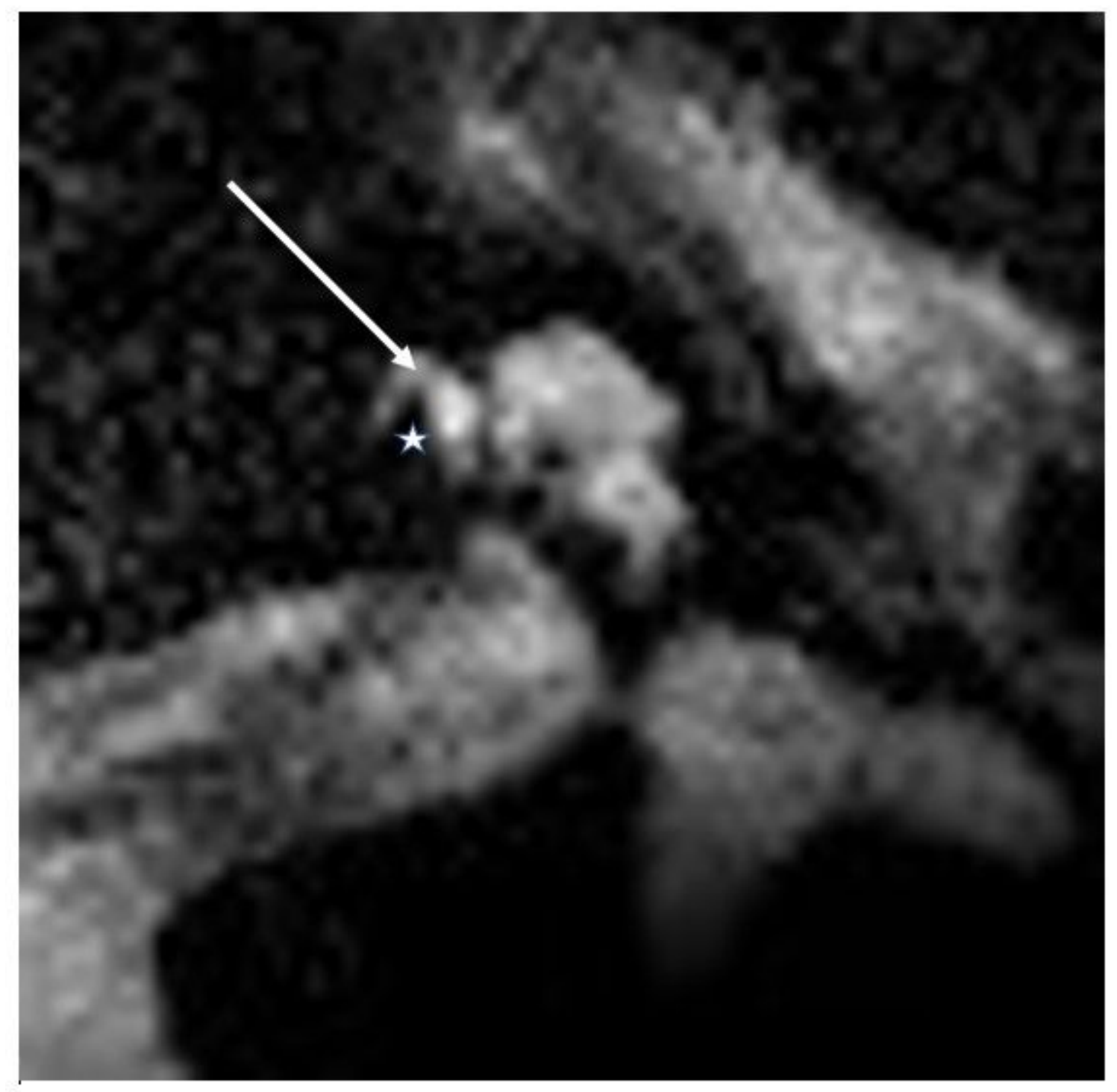

Figure 3

Modiolar Electrode, Basal Turn, HFMS Star represents diminishing electrode signal, arrow represents basilar membrane. 


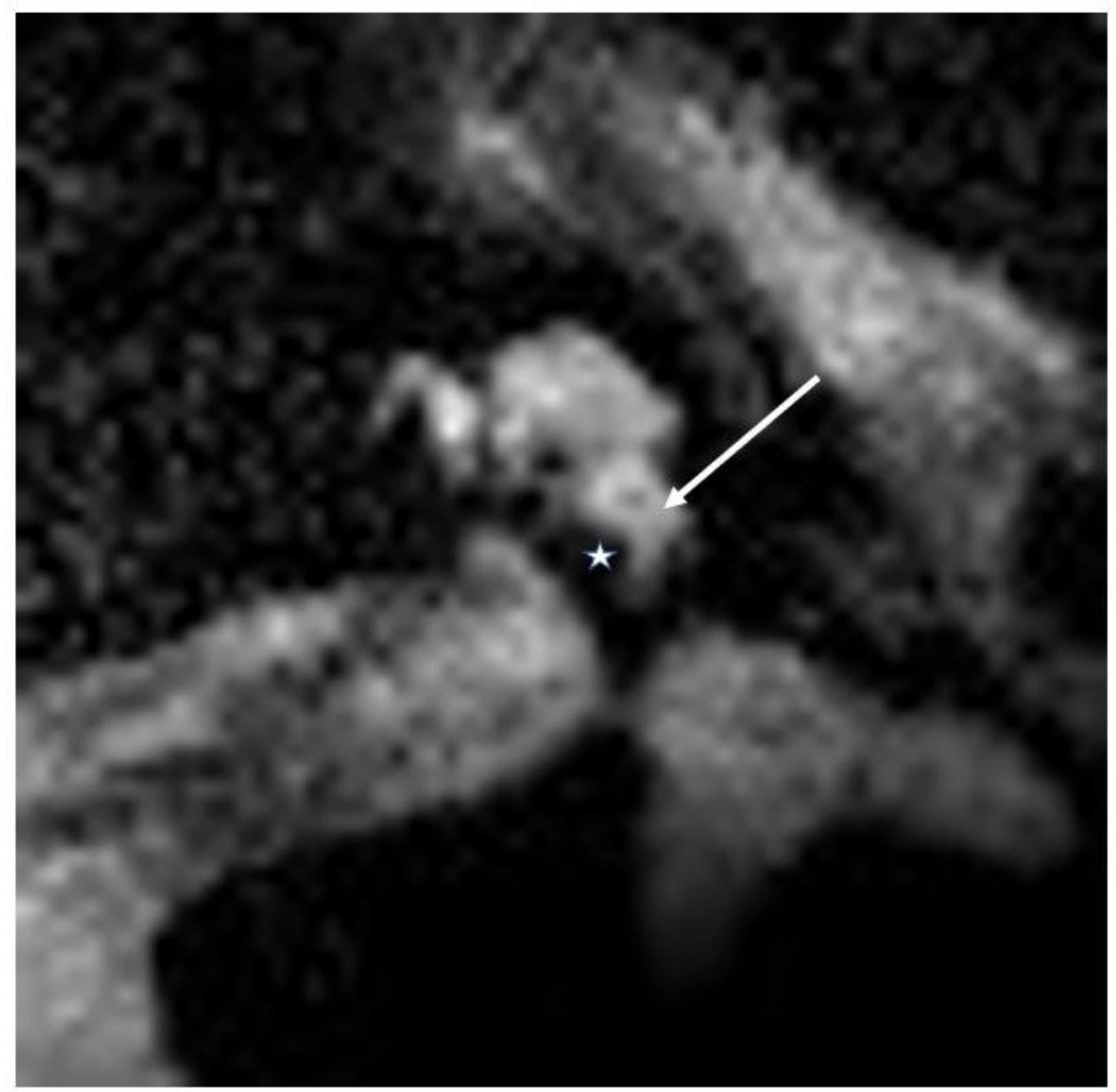

Figure 4

Modiolar Electrode, First Turn, HFMS ST Position Star represents diminishing electrode signal, arrow represents basilar membrane. 


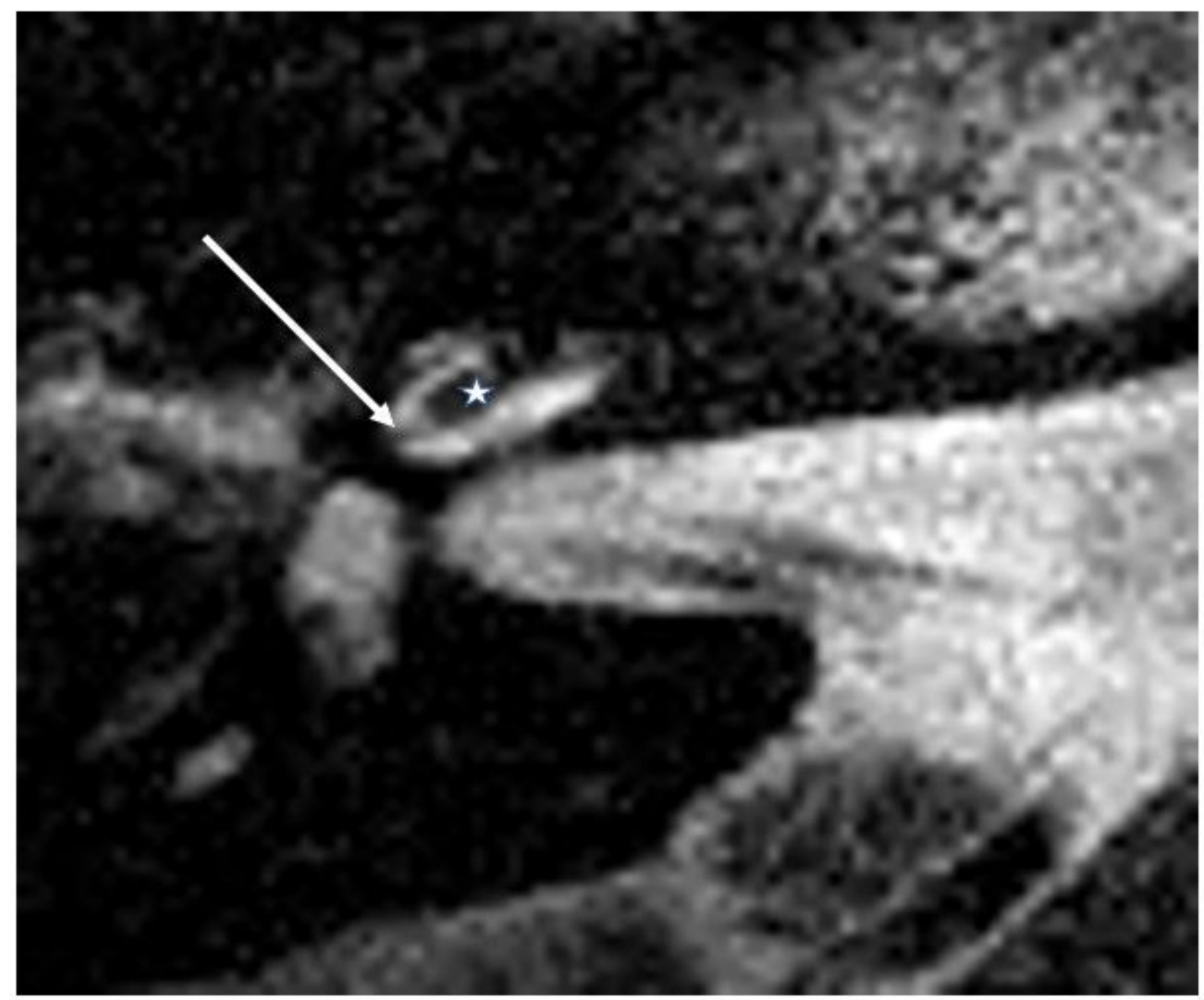

\section{Figure 5}

Modiolar Electrode, First Turn, HFMS SV Position Star represents diminishing electrode signal, arrow represents basilar membrane. Pat.No.10

(A)

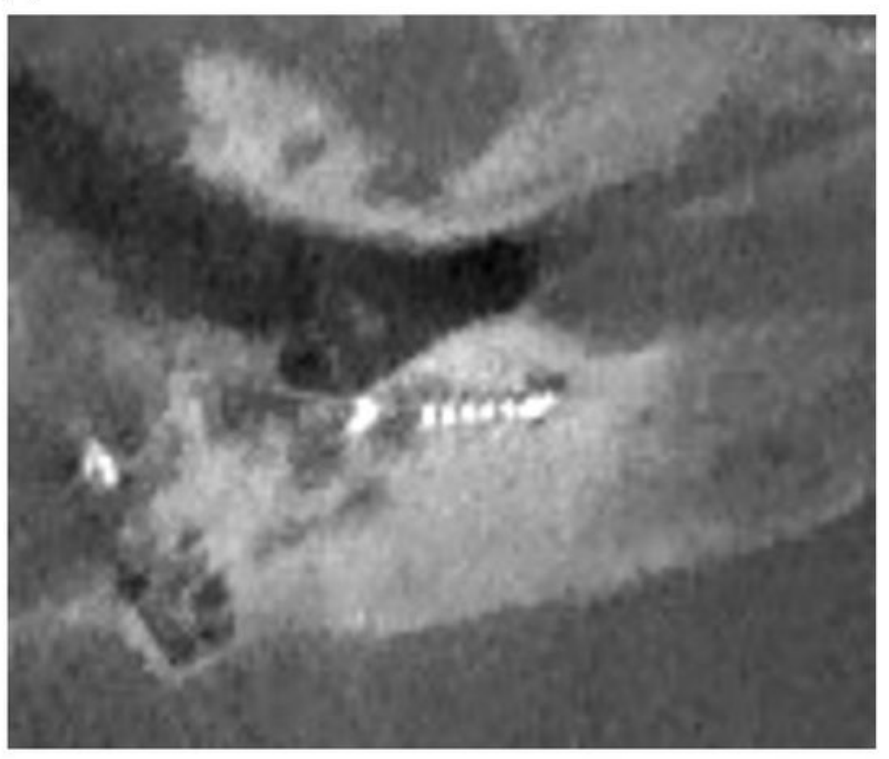

(B)

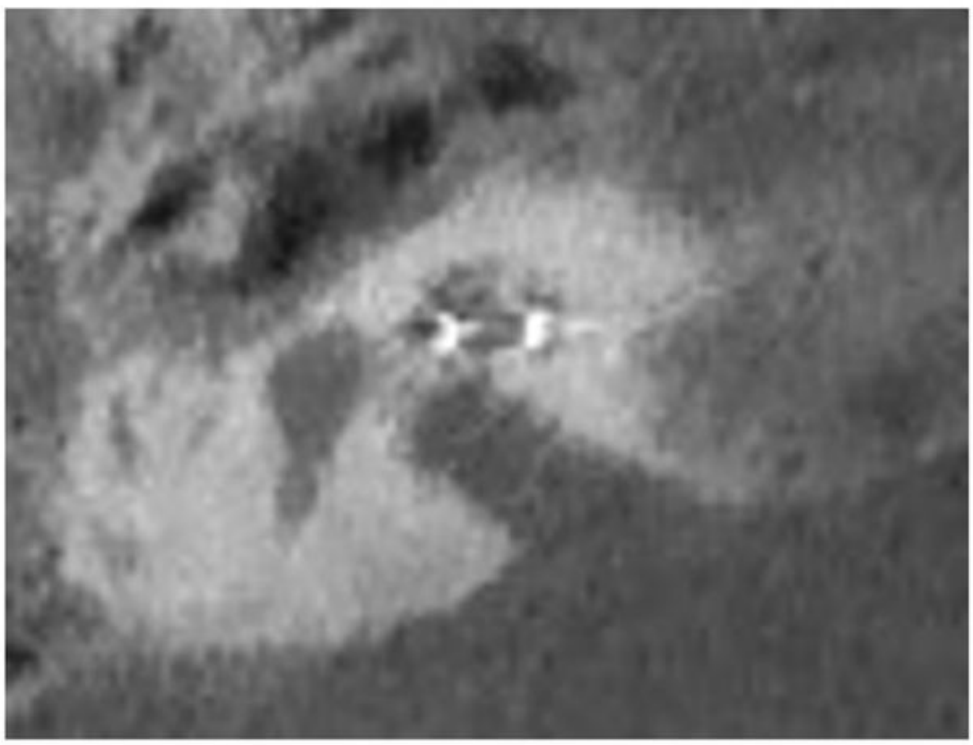




\section{Figure 6}

Exemplary DVT estimated electrode position in the Scala tympani. A) basal turn, b) first turn at the floor of the cochlea.

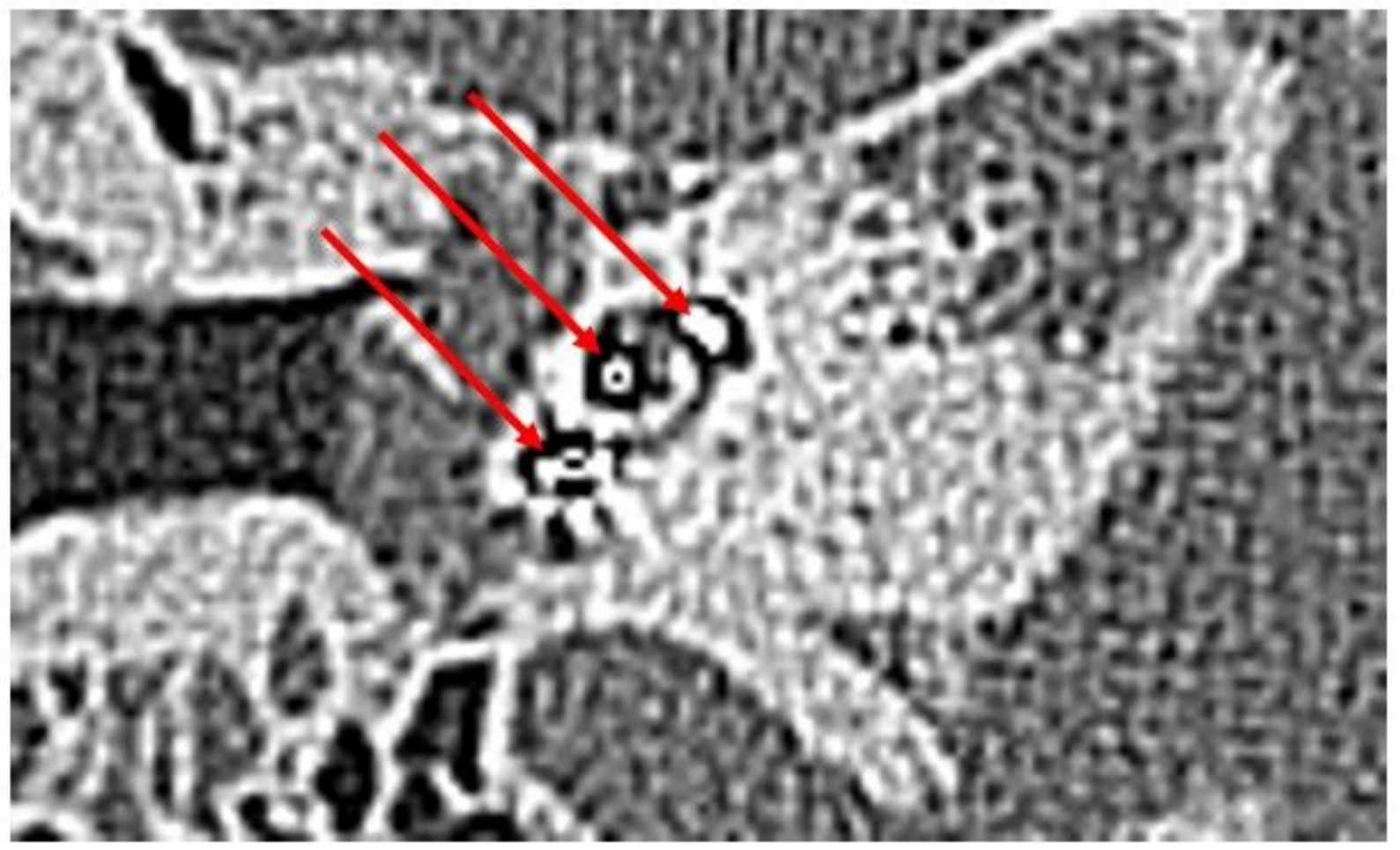

Figure 7

Translocated electrode Arrows show electrode in the basal and first turn. MSCT of Pat. No.10 